Relations industrielles

Industrial Relations

\title{
La sécurité sociale
}

Quand nous serons vieux...

\section{Gérard Tremblay}

Volume 6, numéro 2, mars 1951

URI : https://id.erudit.org/iderudit/1023230ar

DOI : https://doi.org/10.7202/1023230ar

Aller au sommaire du numéro

\section{Éditeur(s)}

Département des relations industrielles de l’Université Laval

ISSN

0034-379X (imprimé)

1703-8138 (numérique)

Découvrir la revue

Citer cet article

Tremblay, G. (1951). La sécurité sociale : quand nous serons vieux... Relations industrielles / Industrial Relations, 6(2), 35-41. https://doi.org/10.7202/1023230ar

Tous droits réservés @ C Département des relations industrielles de l’Université Laval, 1951
Ce document est protégé par la loi sur le droit d'auteur. L'utilisation des services d'Érudit (y compris la reproduction) est assujettie à sa politique d'utilisation que vous pouvez consulter en ligne.

https://apropos.erudit.org/fr/usagers/politique-dutilisation/ 


\section{Quand nous serons vieux....}

par Gérard Tremblay, directeur du Département des relations industrielles de Laval

La sécurité sociale est l'une des premières préoccupations de la civilisation contemporaine. Son champ d'application est vaste comme tous les risques sociaux qu'elle entend couvrir.

Même ici au Canada, un élément important de la sécurité sociale, à savoir, l'assurance et l'assistance vieillesse, a pris la vedette, et, n'eût été les événements de Corée dont la portée internationale n'échappe à personne, la question serait l'une des premières à l'ordre du jour.

Nous croyons intéresser nos lecteurs en donnant une rétrospective du rapport de la Commission mixte d'Enquête du Sénat et de la Chambre des Communes sur la sécurité du vieil âge et en analysant à grands traits les conclusions du Comité lui-même et les vues exprimées par les principales organisations ouvrières et patronales qui ont jugé à propos de comparaître devant ses membres.

Il importe de définir la «sécurité sociale». Sir William Beveridge lui donne la signification suivante:

« Le terme « sécurité sociale \ implique la garantíe d'un revenu qui puisse remplacer les gains lorsque ceux-ci sont interrompus du fait du chômage, de la maladie ou de l'accident; qui doive assurer la subsistance à raison de la retraite due à la vieillesse, du décès, du soutien d'une autre personne et qui puisse permettre de rencontrer des dépenses exceptionnelles telles que celles qu'occasionnent la naissance, la mort et le mariage. $\$ 1$

Dans un cours que nous avons professé à l'Université, nous avions nousmême défini la «sécurité sociale»

(1) Report by Sir William Beveridge, The MacMillan Company, New York, 1942. de façon subjective comme un état de confiance et de tranquillité d'esprit des masses populaires résultant de la pensée qu'elles n'ont pas à craindre, pour le moment ou pour l'avenir, en raison de mesures d'assurance ou d'assistance sociale appropriées, les misères d'ordre économique provenant de certains maux et risques -sociaux qui entraînent une perte ou une diminution de revenu, tels que le chômage, la maladie, les accidents, l'invalidité, la vieillesse, les charges exceptionnelles de famille et la mort.

Elle peut être aussi définie brièvement comme étant la garantie des moyens d'existence perdus en raison de l'incapacité de travailler ou d'obtenir un emploi.

\section{Le rapport}

Nous entendons limiter notre étude à la couverture d'un seul risque, celui de la « vieillesse * qui, du reste, a fait l'objet des travaux de la Commission mixte d'Enquête du Sénat et de la Chambre des Communes.

La Commission s'est vu confier la lourde responsabilité de suggérer le système de pensions au vieil âge le mieux approprié à nos conditions sociales et aux exigences de l'économie canadienne. Il a reçu des mémoires préparés par les provinces et les principales organisations patronales, ouvrières et sociales. Bénéficiant d'études déjà faites par les services administratifs compétents, la Commission a été en mesure, pendant trois mois, soit du début d'avril au 28 juin, 1950, de compulser 1,300 pages de rapports de tous genres, sans compter l'audition des témoignages oraux. Travail immense et qui mon- 
tre que notre démocratie canadienne est capable, par le truchement de comités d'étude sérieux, d'exécuter de la bonne besogne.

Nous nous bornerons à résumer les conclusions du Comité en nous inspirant du rapport officiel. ${ }^{2}$

Le Comité propose en bref le plan suivant qui, on le sait, a été soumis aux délégués à la conférence fédérale-provinciale de l'automne dernier. Ce plan est le fruit de la juxtaposition de deux systèmes, le premier s'inspirant du régime de l'assurance, le second de celui de l'assistance:

a) Un système universel de pensions de vieillesse financé au fur et ì mesure pour toutes les personnes âgées de 70 ans et plus, à base de cotisations, et dont l'administration serait confiée au gouvernement fédéral. La prestation serait d'un montant fixe et uniforme de $\$ 40$ par mois pour tous les individus admissibles, et il n'y aurait que deux conditions d'admissibilité: l'âge et une période raisonnable de résidence au Canada;

b) Quant aux personnes âgées de 65 ans ou plus, non admissibles à toucher la pension universelle, un système d'assurance-vieillesse avec allocation maximum de $\$ 40$ par mois serait établi. Cette allocation serait accordée subordonnément à une épreuve d'admissibilité assez semblable à celle qui existe en vertu du présent programme d'assistance - vieillesse, mais modifiée de manière à tenir compte des caractéristiques du groupe d'âge en question. Il faudrait aussi corriger cette épreuve de façon que les allocataires soient encouragés plus qu'ils ne le sont à l'heure actuelle à gagner un supplément de revenu.

\section{Assurance}

Le régime spécial prévu par ce plan entraînerait un déboursé annuel de $\$ 323,760,000$. Il est basé sur l'admission qu'en $1951,674,500$ personnes de 70 ans et plus recevraient une pension mensuelle de $\$ \mathbf{4 0}$. A noter qu'en

(2) Rapport du Comité mixte d'Enquête du Sénat et de la Chanbre des Communes sur les pensions de vieillesse. régime d'assurance, tous les citoyens auraient droit à la prestation sans évaluation de leurs ressources pour l'excellente raison qu'ils auraient contribué à alimenter de leurs contributions la caisse générale.

Ces contributions dans le cas des salariés seraient payées par eux-mêmes et leurs employeurs; dans le cas des personnes non-salariés, à même leurs revenus. Il est admis que l'Etat fédéral ferait les avances nécessaires pour le compte des personnes dans l'impossibilité de contribuer.

Il ne saurait être question de constituer un fonds de réserve d'après les données mathématiques que suivent les actuaires d'assurance-vie. Il faudrait maintenir alors pendant des années le système actuel d'assistance pour les vieux qui n'ont pas eu l'opportunité d'accumuler un capital assez fort pour garantir une rente annuelle de $\$ 480$. Le rapport indique qu'à 70 ans, un homme devrait avoir à son crédit un capital de $\$ 4,690$ et une femme, de $\$ 5,500$ pour garantir le service des prestations. On imagine l'immobilisation fantastique des capitaux requis mand les statistiques révèlent qu'il $y$ a au pays en 1951 674,500 personnes de 70 ans et plus.

On convient, par ailleurs, que les bénéficiaires en raison du manque à gagner, ne seraient pas toujours en mesure de payer régulièrement leurs primes. De là l'accumulation d'un capital insuffisant pour assurer le paiement des prestations minima.

On a donc recours au système universel de prestations financé au fur et à mesure. Les cotisations payées sous forme de taxes, soit par les salariés, soit par les employeurs ou soit encore par un prélèvement sur le revenu, ne conditionnent pas nécessairement le versement des prestations. Elles sont suffisantes cependant, avec la mise de l'Etat, pour assurer leur paiement d'année en année. Tous les vieillards de 70 ans et plus sont donc assurés du paiement de la pension. Dans quelques années, presque tous les bénéficiaires pourront se dire 
qu'ils ont contribué à leur retraite. Cest une facon indirecte de les dégager de l'odieux que peut avoir, au point de vue psychologique, l'enquête sur l'évaluation de leurs besoins et le bénéfice d'une pension qui a couleur de charité publiaue.

Le gouvernement fédéral devrait lui-même constituer l'organisme de perception et de gestion de l'assurance étant donné la nécessité d'établir celle-ci sur une base nationale, permettant ainsi au citoyen de paver ses primes ou de recevoir ses prestations en tout endroit du pays. De là, nécessité d'amender la constitution canadienne pour autoriser l'autorité fédérale à administrer l'assurance-vieillesse comme on l'a fait lors de la mise en vigueur de l'assurance-chômage.

Le Comité ne recommande pas l'extension du système aux personnes de 65 à 70 ans pour cette raison que des ressources supplémentaïres de $\$ 204,000,000$. devraient être recueillies des assurés et des contribuables portant ainsi au total de $\$ 528,000,000$. le coût national de cette forme particulière d'assurance. Le Comité se demande si le pays, au seul titre des « pensions de vieillesse 》 est disposé à prélever un aussi fort montant sur un revenu national de treize milliards.

\section{Asisistance}

Des pressions constantes ont été faites par les organisations ouvrières et par les sociétés de bienfaisance sociale pour que la pension soit payée aux vieux de 65 à 70 ans. Un grand nombre de citoyens ne trouvent plus preneur de leurs services à cette période de la vie. Bien rares sont ceux qui ont pu acquérir une certaine indépendance financière. La charité publique doit alors, à défaut de la famille, subvenir aux besoins de ces miséreux.

Le Comité propose d'établir un système d'assistance-vieillesse d̀ peu près similaire à celui que nous avons depuis 1927. Allocation mensuelle maximum de $\$ 40$. Vérification de l'évaluation des besoins. Beaucoup de personnes de 65 à 70 ans sont encore en mesure de travailler au moins à temps partiel; d'autres, moins nombreuses, ont accumulé un petit capital leur rapportant une petite rente. L'assistance tiendrait compte de ces revenus et $y$ proportionnerait le paiement complémentaire de la pension.

Quels seront les déboursés de ce plan d'assistance ? On les évalue à $\$ 64,000,000$. pour 1951. La moitié en serait payée par le gouvernement fédéral et l'autre par les provinces. Le coût du système actuel (pension à 70 ans avec preuve d'évaluation des besoins) pour l'année financière 195051 , est évalué à 136 millions, la quote-part du fédéral étant de 100 millions et celle des provinces de 36 millions. C'est donc dire que le fardeau financier des provinces serait sensiblement le même: 32 millions par rapport à 36 millions.

A noter que le plan d'assistance aux vieillards indigents est complémentaire du plan d'assurance-universelle. Ou plutôt, il le précède dans le temps car le vieillard plus ou moins nécessiteux en bénéficiera de 65 d 70 ans pour devenir ensuite titulaire de plein droit, sans évaluation de ressources, de l'assurance nationale de 70 ans à sa mort.

On ne pourra nier le réalisme et la psychologie qu'ont montrés les membres du Comité en intégrant la formule d'assistance à celle de l'assurance en deux stades successifs. Il fallait tenir compte que dans un pays surtout industriel, les personnes âgées de 65 ans sont souvent dans le dénûment; que par ailleurs d'autres, en grand nombre, sont en mesure de travailler au moins à temps réduit ou encore possèdent quelques biens. II ne fallait donc pas du point de vue psychologique et même économique détruire la volonté de travail, la tendance naturelle à l'épargne non plus que la fierté d'une certaine indépendance financière. Il convenait égale- 
ment d'obérer le moins possible le budget national.

$\mathrm{Si}$, par ailleurs, une prestation de $\$ 40$ est payable aux vieillards de 70 ans et plus avec le plan d'assurance, il faut noter que déjà sous le système actuel, 76.3 pour cent de cette catégorie de vieillards reçoivent la pension complète ou partielle. Le fardeau financier ne sera pas tellement accru. Le pourcentage de 23.7 sera sans doute ajouté au nombre des bénéficiaires mais il faut retenir, d'une part, que ces bénéficiaires auront contribué au fonds commun et que, d'autre part, limpôt sur le revenu saura faire rentrer les prestations versées à ceux qui, étant donné leurs revenus, n'en ont pas besoin.

\section{Conditions de résidence}

Quelles sont les conditions relatives à la résidence recommandées par le Comité ? Selon la formule d'assurance universelle à 70 ans, les bénéficiaires citoyens canadiens ou non, doivent avoir résidé au Canada au moins 20 ans; c'est la condition déjà requise par le système actuel de l'assistance fédérale-provinciale.

Quant à la formule d'assistancevieillesse, elle soumet que la durée de résidence au pays devrait se limiter à 15 ans. Pourquoi a-t-on abaissé de 20 à 15 ans la condition de résidence au pays, car en somme le système d'assistance-vieillesse de 65 à 70 ans est la répétition du présent régime d'assistance à 70 ans et plus ? Il semble qu'on ait voulu tenir compte des besoins futurs des nombreux immigrants que nous accueillons depuis la deuxième guerre mondiale. C'est un attrait supplémentaire du pays que de garantir à ses ressortissants indigents une pension mensuelle de $\$ 40$ après seulement 15 années de résidence.

\section{Les initiatives privées}

Il ne faut pas sous-estimer les résultats obtenus à date par l'initiative privée dans la couverture du ris- que-vieillesse. Le rapport de la Commission mixte d'Enquête fournit des informations précises à ce sujet. Sur un total de 5 millions de salariés ${ }^{3}$, près d'un million bénéficient d'un droit à une certaine retraite.

Voici un résumé statistique à ce sujet ${ }^{4}$. En mars $1950,258,600$ personnes détenaient un titre aux rentes viagères prévues par la Loi des Rentes sur l'Etat de 1908. Les primes versées pour 1949-50 étaient de $\$ 63.1$ millions et les rentes servies de $\$ 23.4$ millions. Le maximum de la rente individuelle est de $\$ 1,200$ par année.

En mars 1950, 122,000 syndiqués bénéficiaient de rentes différées en vertu de 846 contrats collectifs. 8,000 employés participaient à 300 plans de pension de retraite prévus par des contrats particuliers.

Des plans de pension pour employés acceptés par le Ministère du Revenu national se totalisaient à la même date à 4,125 couvrant 627,000 salariés.

Le total des bénéficiaires se chiffre donc à $1,015,600$.

Initiative excellente qu'il faut maintenir mais qui, aux yeux des actuaires, a un double défaut. Elle tend à développer chez les employeurs le souci de n'embaucher que des personnes relativement jeunes et elle géne la mobilité de la main-d'oeuvre. Les organismes responsables de. vraient trouver les moyens de remédier à ces graves inconvénients pour les individus et pour l'économie na. tionale.

Il ne faudrait pas ignorer non plus les millions d'assurés bénéficiaires de plan d'assurance-vie individuelle: vie entière, vie primes limitées, vie dotation, etc. qui ont su prévoir pour l'avenir, bien avant qu'au nom de la sécurité sociale, on ait commencé à

(3) Circular No. 2230. Canadian Manufacturers' Association.

(4) Voir Rapport du Comité, pp. 12 et suivantes.

La Revue des relations industrielles 
se préoccuper du problème de la sécurité personnelle. ${ }^{5}$

\section{Plan de la Confédération des Travailleurs Catholiques du Canada ${ }^{\circ}$}

Les organisations ouvrières ont présenté des inémoires à la Commis. sion mixte d'Enquête. Nous croyons utile de les résumer. Le projet de la C.T.C.C. comporte:

1-Une pension de $\$ 50$ par mois aux femmes ayant atteint l'âge de 60 ans et aux hommes ayant atteint l'àge de 65 ans, en vertu d'un régime unjversel de pensions de vieillesse, dont le coût serait défrayé aux trois-cinquièmes par le gouvernement fédéral, et aux deux-cinquièmes par les provinces;

2-La fixation pour les allocataires d'une période raisonnable de résidence au Canada;

3-L'abolition des enquêtes sur les moyens de subsistance (means test);

4-L'établissement en faveur des salariés d'une assurance contributoire, avec caisse de réserve, en vue d'une pension de retraite de $\$ 50$ par mois à l'âge de 60 ans pour les femmes et de 65 ans pour les hommes. Les salariés verseraient deux pour cent $(2 \%)$ de leur salaire à la caisse de réserve; les employeurs paieraient la balance nécessaire pour assurer la solvabilité de la caisse; les gouvernements assumeraient les frais d'administration et combleraient les défcits;

5-La protection des régimes collectifs existants de pensions de retraite et. au moyen de négociations collectives, l'établissement d'autres plans comme compléments des régimes plus haut suggérés;

6-La constitution d'organismes appropriés pour surveiller l'application de la loi et assurer le paiement des pensions aux personnes qui $y$ ont droit.

(5) La Canadian Life Insurance Officers Ass'n a rapporté que 61 sociétés affiliées détenaient 14 milliards et demi d'assurancevie sur 5 millions de Canadiens et cela à l'exclusion des sociétés fraternelles de tous genres (vide p. 65, Idem).

(6) Mémoire annuel de la C.T.C.C. au Cabinet provincial, 1951. Rapport du président sur la Sécurité sociale, au Congrès de la C.T.C.C., Sherbrooke, 1950.
Il importe de connaitre le coùt approximatif du projet. La pension (assistance) de $\$ 50$ par mois versée a 490,000 femmes de 60 ans et plus et à 400,000 hommes de 65 ans et plus coûterait $\$ 900$ millions.

La pension (assurance) destinée à augmenter de $\$ 50$ à $\$ 100$ le revenu mensuel total d'une femme de 60 ans et diun homme de 65 ans coûterait une prime totale d'à peu près $10 \%$ (salarié $2 \%$, employeur $6 \%$, Etat $2 \%$ ). Sur la faible base d'un salaire annuel moyen de $\$ 1,500$, il faut somme toute songer pour 5 millions de salariés à des cotisations tripartites additionnelles rapportant près de $\$ 900$ millions. Caisse de réserve prévue, selon le plan.

Ajoutez à cela le maintien des régimes collectifs existants dont le coût pour un million de salariés représente une immobilisation d'à peu près $\$ 150$ millions.

Nous obtenons ainsi un grand total d'obligations annuelles de près de 2 milliards au seul titre de la retraite, sans prévoir conséquemment pour les autres éléments de la sécurité sociale. Et cela par rapport à un revenu national de 13 milliards.

\section{Plan du Congrès des Métiers et du Travail (Fédération Américaine du Travail)}

Le Congrès des Métiers et du Travail, dans son mémoire, a soumis que le système actuel des pensions de vieillesse devrait être bonifié. Il se réfère d'abord à sa proposition de mars 1950 à l'effet que l'allocation mensuelle soit portée de $\$ 40$ à $\$ 60$ et que l'âge d'admissibilité soit abaissé de 70 à 65 ans pour les hommes et à 60 ans pour les femmes.

Le coût approximatif du plan proposé serait de $\$ 975$ millions.

Dans sa présentation spéciale au Comité, le Congrès limite cependant sa demande à une mensualité de $\$ 60$ payable uniformément aux vieillards 
des deux sexes à l'âge de 65 ans (la proposition d'une pension d 60 ans pour les femmes n'est pas retenue). Le coût en serait de $\$ 973$ millions. Une résidence de 15 ans ouvrirait un droit à la prestation qui ne serait pas conditionnée par l'évaluation des ressources.

Les vues exprimées par le Congrès onit ceci d'intéressant qu'elles se déclarent ouvertement en faveur d'un système de sécurité sociale universelle et contributoire qui couvrirait en conséquence les risques chômage, vieillesse, maladie et mères nécessiteuses. ?

\section{Plan du Congrès Canadien du Travail (Congrès des organisations industrielles)}

Quant au Congrès Canadien du Travail, il réclame également l'organisation complète de la sécurité sociale. Son mémoire au Comité d'Enquête recommande que la pension mensuelle soit acorue de $\$ 40$ à $\$ 50$ et que l'âge d'admission soit abaissé à 65 ans, sans évaluation de ressources. Le coût annuel du plan atteindrait $\$ 660$ millions. Il serait financé au fur et à mesure par un ajustement de l'impôt sur le revenu, le rétablissement de l'impôt sur les corporations, l'augmentation de $25 \%$ de l'impôt sur le revenu des particuliers et l'abaissement du niveau d'exemption des salariés pour fin expresse de financement des pensions de vieillesse.

Tout comme la Confédération des Travailleurs Catholiques du Canada, le Congrès Canadien du Travail est favorable à l'organisation d'une assurance complémentaire à l'assistance universelle, avec cette particuliarité qu'elle établirait des cotisations et des allocations graduées d'après le revenu. L'allocation ne devrait pas excéder $\$ 50$ par mois de façon à ce

(7) Trades and Labor Congress of Canada, 65th Annual Convention, Montreal 1950. que les pensions d'assistance et d'assurance réunies ne dépassent pas $\$ 100$ par mois.

Un aspect original de la proposition du Congrès Canadien du Travail est que la pension de base de $\$ 50$ devrait s augnenter curnulativement de 2 pour cent par année, cette plusvalue étant basée sur l'augmentation continuelle de la productivité nationale. Ce qui revient à dire que dans dix ans la pension de base serait haussée à $\$ 60$ par mois du fait de son accroissement correspondant de 20 pour cent durant la même période.

\section{Plan de l'Association des Manufacturiers Canadiens}

Quelles sont les propositions du groupe patronal ? $\mathrm{La}$ plus intéressante est celle de l'Association des Manufacturiers Canadiens (C.M.A.). Elle suggère l'assurance contributoire tripartite, employeurs et salariès payant à part égale les cotisations et l'Etat assurant le paiement des frais d'administration. L'âge d'admission à la retraite serait fixé entre 65 et 70 ans en tenant compte du principe que beaucoup de travailleurs sont en mesure entre ces deux points d'âge de gagner quelque revenu.

Quant au montant de la retraite, l'Association estime qu'il devrait varier en raison des cotisations fournies, avec garantie d'un minimum raisonnable pour ceux qui n'ont pu contribuer suffisamment. Il va de soi que le système d'assistance fédéraleprovinciale actuel serait continué pour les personnes trop âgées ou incapables de participer financièrement au plan. Dans la mesure où le fonds d'assurance interviendrait, s'abaisserait aussi le fardeau de l'assistance.

A noter que l'Association recommande à la fois une exemption du plan pour les personnes à revenus trop modestes ou trop élevés. 


\section{Conclusions}

Il semble assuré que le gouvernement fédéral introduira, cette année, un bill en vue de donner suite aux recommandations du Comité mixte d'Enquête du Sénat et de la Chambre des Communes. Par ailleurs, le chef du gouvernement de notre province vient d'annoncer qu'un bill sera présenté à la législature aux fins d'autoriser la négociation d'accords avec le gouvernement canadien pour l'organisation d'un système modifié des pensions de vieillesse. Se pose aussi la question d'un amendement à la constitution pour la mise en vigueur d'une assurance financée au fur et à mesure. On se rappelle, en effet, que le Comité a recommandé l'assurance universelle et le service des prestations de plein droit aux vieillards de 70 ans et plus. Quant au régime complémentaire d'assistance aux personnes indigentes de 65 à 70 ans, c'est la transposition du système déjà en vigueur pour les vieillards de 70 ans et plus; pas besoin dans ce dernier cas d'amender la constitution, il suffit de refaire les ententes fédéraleprovinciale.

Le système proposé est-il le meilleur? Nous ne le croyons pas. Nous nous rangerions plutôt en faveur des éléments essentiels des projets mis de l'avant par le Congrès Canadien du Travail et l'Association des Manufacturiers Canadiens. Organisation de l'assurance contributoire à cotisations et à prestations graduées. Rien de plus juste. Un ouvrier qualifié et un employé de grade moyen sont en mesure de verser, à même leur revenu, une cotisation supérieure. Pourquoi ne recevraient-ils pas une prestation proportionnellement plus élevée ? Il y a là un élément d'émulation légitime pour tous les citoyens à se hausser dans une catégorie où la retraite garantira un plus grand confort et une sécurité plus complète.

Par ailleurs, le Congrès Canadien du Travail comme l'Association des Manufacturiers Canadiens sont favorables, selon un réalisme de bon aloi, au maintien de l'assistance minimum pour tous les vieillards.

L'objection que le Comité a soulevée contre l'assurance graduée est la complication des opérations comptables. Nous ne croyons pas qu'il y ait là un argument solide quand on songe à la haute technique que les percepteurs de l'impôt sur le revenu ont su développer.

En dernière analyse, les quelques mois à venir et l'expérience des prochaines années nous diront ce que nous recevrons lorsque nous serons devenus vieux. Il importe avant de poser le point final à cet article de mettre nos législateurs en garde contre la tendance à niveler les prestations tout en graduant les cotisations directes ou indirectes. La sécurité sociale est excellente mais à condition que l'état du citoyen moyen ne soit pas abaissé à la commune mesure de lindigence.

Il faut craindre également la main mise graduelle d'un Etat de plus en plus omnipotent sur la vie des citoyens; on pourrait peut-être, mais trop tard, se rendre compte qu'une sécurité miséreuse a été payée par la monnaie d'or de la liberté.
Si le maitre traite les ouvriers, non comme des hommes, mais comme des instruments de production, il arrive facilement qu'ils cessent d'étre des hommes.

\section{Harmel}

(Expérience du Val des Bols)
Celui qui ne sait donner sa confiance qu'aus compte-gouttes ne doit pas s'attendre d recevoir le dévouement avec une pompe d incendie.

Rozack (L'offrande à Mercure) 